\title{
DETECTION OF ENDOGENOUS TISSUE FACTOR LEVELS IN PLASMA USING THE CALIBRATED AUTOMATED THROMBOGRAM ASSAY
}

\author{
Veronique Ollivier ${ }^{1}$, Jianguo Wang ${ }^{2}$, David Manly $^{2}$, Kellie R. Machlus $^{3}$, Alisa S. Wolberg ${ }^{3}$, \\ Martine Jandrot-Perrus ${ }^{1}$, and Nigel Mackman ${ }^{2}$ \\ ${ }^{1}$ Inserm, U698, Paris, F-75018 France; Université Paris 7, Paris, F-75018 France. \\ ${ }^{2}$ Division of Hematology/Oncology Department of Medicine University of North Carolina at Chapel \\ Hill Chapel Hill, North Carolina, USA \\ ${ }^{3}$ Department of Pathology University of North Carolina at Chapel Hill Chapel Hill, North Carolina, \\ USA
}

\section{Summary}

Background-The calibrated automated thrombogram (CAT) assay measures thrombin generation in plasma.

Objective-Use the CAT assay to detect endogenous tissue factor (TF) in recalcified platelet-rich plasma (PRP) and platelet-free plasma (PFP).

Methods-Blood from healthy volunteers was collected into citrate and incubated at $37^{\circ} \mathrm{C}$ with or without lipopolysaccharide (LPS) for 5 hours. PRP and PFP were prepared and clotting was initiated by recalcification. Thrombin generation was measured using the CAT assay.

Results-The lag time (LT) was significantly shortened in PRP prepared from LPS-treated blood compared with untreated blood ( $10 \pm 3$ min versus $20 \pm 6 \mathrm{~min})$, and this change was reversed by the addition of inactivated human factor VIIa. LPS stimulation did not change the peak thrombin. Similar results were observed in PFP ( $21 \pm 4$ min versus $35 \pm 5 \mathrm{~min})$. LPS stimulation also significantly reduced the LT of PRP and PFP derived from blood containing citrate and a factor XIIa inhibitor. Finally, a low concentration of exogenous TF shortened the LT of PFP prepared from unstimulated, citrated blood without affecting the peak thrombin.

Conclusion-Changes in LT in the CAT assay can be used to monitor levels of endogenous TF in citrated plasma.

\section{Keywords}

tissue factor; thrombin; plasma; platelets

(C) 2009 Elsevier Ltd. All rights reserved.

Corresponding author: Nigel Mackman, PhD John Parker Professor of Medicine Division of Hematology/Oncology Department of Medicine University of North Carolina at Chapel Hill Chapel Hill, North Carolina Tel 9198433961 Fax 9199667639 Email: nmackman@med.unc.edu.

Publisher's Disclaimer: This is a PDF file of an unedited manuscript that has been accepted for publication. As a service to our customers we are providing this early version of the manuscript. The manuscript will undergo copyediting, typesetting, and review of the resulting proof before it is published in its final citable form. Please note that during the production process errors may be discovered which could affect the content, and all legal disclaimers that apply to the journal pertain. 


\section{Introduction}

Tissue factor (TF) is a transmembrane glycoprotein that is essential for hemostasis (1-3). Extravascular cells constitutively express TF to form a primary hemostatic barrier around blood vessels (4). Following vessel injury TF activates the coagulation cascade leading to thrombin generation, fibrin deposition and platelet activation. In pathological conditions, such as endotoxemia and sepsis, TF is expressed by circulating monocytes (5-8). Indeed, several studies have shown that bacterial lipopolysaccharide (LPS) induces TF expression in monocytes and monocytic cells (4) (9-12). TF is also found in plasma in the form of microparticles (MPs), which are small $(<1 \mu \mathrm{M})$ membrane vesicles generated by activated or apoptotic cells (13). TF-positive MPs may contribute to thrombosis in patients with a variety of diseases, including cardiovascular disease, sickle cell disease, endotoxemia and cancer (14-23).

The level of TF in plasma can be measured using various methods. Enzyme-linked immunosorbant assays and flow cytometry measure TF antigen (18) (24-27); however, because antibodies may recognize "encrypted" TF or degraded forms of TF that are not active, levels of TF antigen may not correlate with procoagulant activity. Recently, two groups have described assays that measure MP TF activity (16) (19). One study captured the TF-positive MPs with an antibody and showed that infusion of LPS into volunteers increased MP TF activity (16). Another study isolated MPs by centrifugation and showed that patients with advanced cancer had elevated levels of MP TF activity (19). We recently developed a similar MP TF activity assay (28). It is worth noting, however, that these activity assays require multiple steps and are time-consuming.

Hemker and colleagues developed the Calibrated Automated Thrombogram (CAT) assay to measure thrombin generation in plasma (29). In this assay, thrombin generation is triggered by the addition of low levels of recombinant, relipidated tissue factor or endogenous procoagulant MPs (30-32). Thrombin generation is monitored via cleavage of a fluorogenic substrate that allows the measurement of thrombin in the presence of fibrinogen and platelets (33). Test samples are normalized for plasma color, inner filter effect and substrate depletion to produce a thrombogram. Lag time (LT) and peak of thrombin generation can be derived from the thrombogram. The CAT assay has been used for a variety of purposes, including detection of clotting factor deficiencies, monitoring of warfarin therapy, and the efficiency of replacement factor therapy in hemophiliacs (30) (34-36). Notably, it has also been used to detect hypercoagulable states, including congenital heart disease, severe sepsis syndrome, acute deep-vein thrombosis and patients at risk of recurrent venous thromboembolism $(37-40)$.

In this study, we examined if the CAT assay could be used to detect changes in endogenous TF levels in platelet-rich plasma (PRP) and platelet-free plasma (PFP). We used a model system of LPS-treated whole blood to generate TF. We found that the presence of endogenous TF in PRP and PFP reduced the LT but did not affect the peak thrombin. These results indicate that the LT in the CAT assay can be used to monitor levels of endogenous TF in plasma.

\section{Materials and methods}

\section{Materials}

LPS (Escherichia coli serotype O111:B4) was obtained from Sigma-Aldrich, St Louis, MO. MP reagent (Diagnostica Stago, Asnières, France) was used as a source of phospholipids and contains phosphatidylserine, phosphatidylcholine and phosphatidylethanolamine at a ratio of 20:60:20. The PRP reagent (Diagnostica Stago) was used as a source of recombinant TF. Corn trypsin inhibitor (CTI) and factor XI were purchased from Haematologic Technologies, Inc 
(Essex Junction, VT). Factor XI-deficient plasma was purchased from HRF, Inc (Raleigh, NC). Human factor VIIa and factor X were purchased from Enzyme Research Laboratories (South Bend, IN). Factor VIIai was obtained from American Diagnostica (Stamford, CT).

\section{Whole blood collection, LPS stimulation and preparation of PRP and PFP}

Venous blood was obtained from healthy consenting volunteers not currently taking any medication. Blood was collected via atraumatic antecubital venipuncture into siliconized Vacutainer ${ }^{\mathrm{TM}}$ tubes (Becton Dickinson, Meyton, France) containing buffered sodium citrate ( $0.129 \mathrm{M}$, nine parts blood to one part sodium citrate solution). The first tube of blood was discarded. In some experiments CTI ( $18.3 \mu \mathrm{g} / \mathrm{ml}$ final concentration) was added to the Vacutainer ${ }^{\mathrm{TM}}$ tubes prior to blood draw (41). Whole blood was incubated at $37^{\circ} \mathrm{C}$ with or without LPS for 5 hours. After incubation, whole blood was centrifuged at $200 \mathrm{~g}$ for $15 \mathrm{~min}$ at room temperature to prepare PRP. Platelet counts in PRP were adjusted to150,000 platelets/ $\mu \mathrm{l}$ (final) by dilution with autologous PFP. PFP was prepared by centrifuging PRP at $2000 \mathrm{~g}$ for $15 \mathrm{~min}$ followed by a $13,000 \mathrm{~g}$ spin for 2 min to remove any remaining platelets. MPdepleted plasma was prepared by centrifuging PFP at 20,000g for $15 \mathrm{~min}$. Thrombin generation was measured in freshly prepared PRP and PFP.

\section{Thrombin generation}

Thrombin generation was measured using a Thrombinoscope (Thrombinoscope BV, Maastricht, Netherlands). Briefly, $80 \mu \mathrm{l}$ of each sample was mixed with $20 \mu \mathrm{l}$ of HEPES buffered-saline (pH 7.35, $20 \mathrm{mM}$ HEPES, $140 \mathrm{mM} \mathrm{NaCl}, 5 \mathrm{mg} / \mathrm{ml}$ bovine serum albumin). Samples spiked with $20 \mu \mathrm{l}$ thrombin calibrator (Diagnostica Stago, Asniéres, France) were run in parallel with each cycle of test sample. Thrombin generation was triggered by addition of $\mathrm{CaCl}_{2}$ (16.7 nM final concentration) and fluorogenic substrate Z-Gly-Gly-Arg-AMC (417 $\mu \mathrm{M}$ final concentration, $\mathrm{pH} 7.5,20 \mathrm{mM}$ HEPES, $60 \mathrm{~g} / \mathrm{l}$ bovine serum albumin). The Thrombinoscope software measures several parameters including LT, peak of thrombin generation and endogenous thrombin potential (ETP), which correlates to the area under the thrombogram curve. The contribution of TF to thrombin generation was assessed by preincubating samples at room temperature for $10 \mathrm{~min}$ with inactivated factor VIIa (FVIIai) $(100 \mathrm{nM})$.

\section{MP TF Activity Assay}

We have recently described a MP TF activity assay (28). Briefly, MPs were isolated from PFP by centrifugation at $20,000 \mathrm{~g}$ for $15 \mathrm{~min}$ at $4{ }^{\circ} \mathrm{C}$, washed twice and resuspended in buffer. Samples were incubated with either an inhibitory anti-human TF monoclonal antibody (HTF1) (42) or a control mouse IgG for $15 \mathrm{~min}$ at room temperature before performing a two-stage chromogenic assay with human factor VIIa and human factor X. TF-dependent factor Xa generation $(\mathrm{pg} / \mathrm{ml})$ was determined by subtracting the amount of factor Xa generated in the presence of HTF1 from the amount of factor Xa generated in the presence of the control antibody.

\section{Statistical analysis}

Data are expressed as mean \pm standard deviation (SD). Differences between experimental conditions were analyzed using a one-tailed Student's $t$-test. A probability value $\mathrm{p}<0.05$ was considered statistically significant. 


\section{Results}

\section{Thrombin generation in PRP and PFP prepared from citrated blood with or without LPS stimulation}

The LT of PRP isolated from LPS-treated blood was significantly shorter than the LT of PRP isolated from blood without LPS stimulation (10 \pm 3 min versus $20 \pm 6 \mathrm{~min}$ ) (Fig. 1A). Addition of FVIIai reversed the LPS-dependent shift in LT indicating that the change was due to the expression of TF. Similar results were observed using an inhibiting anti-human TF antibody called HTF1 $(10 \mu \mathrm{g} / \mathrm{ml}$ ) (data not shown). LPS stimulation did not change the peak of thrombin generation or the ETP in PRP (Fig. 1B and data not shown). LPS treatment also shortened the LT in PFP (21 \pm 4 min versus $35 \pm 5 \mathrm{~min})$, which was reversed by factor VIIai (Fig. $2 \mathrm{~A})$. The peak of thrombin generation was not significantly affected by LPS treatment (Fig. 2B). Of note, levels of thrombin were higher in PRP compared with PFP.

\section{LPS dose response}

Our initial studies used a high dose of LPS to potently stimulate the blood. We next determined if LPS produced a dose-dependent change in the LT. We found that LPS induced a dosedependent shortening of the LT in both PRP and PFP (Fig. 3A and 3B). Consistent with our earlier findings, the peak for both PRP and PFP was not significantly affected by addition of different doses of LPS (data not shown). A significant difference in LT was observed in PRP with lower doses of LPS compared with PFP, which is most likely due to the larger amounts of thrombin generated in PRP.

\section{Role of factor XIla in thrombin generation in PRP and PFP from untreated and LPS-treated blood}

To discern TF triggered thrombin generation from contact activation we compared results from blood collected into tubes containing citrate alone with those using blood collected into tubes containing citrate and CTI, a factor XIIa inhibitor. We found that CTI prolonged the LT in PRP and PFP from unstimulated blood, but did not significantly change the LT in PRP and PFP from LPS-stimulated blood (Fig. 4A and C). Importantly, LPS treatment significantly shortened the LT of PRP and PFP derived from blood containing citrate and CTI (Fig. 4A and C). CTI decreased the peak of thrombin generation in both PRP and PFP from unstimulated and LPS-stimulated blood (Fig. 4B and D). Importantly, LPS treatment significantly shortened the LT of PRP and PFP derived from blood containing citrate and CTI.

\section{Effect of exogenous phospholipids on thrombin generation}

We hypothesized that PFP had a prolonged LT compared with PRP because PFP had a limited amount of phospholipid surface for assembly of the coagulation protein complexes. Therefore, we examined the effect of adding exogenous phospholipids on thrombin generation. Addition of exogenous phospholipids to the PFP caused a dose-dependent reduction of the LT and an increase in the peak of thrombin in both untreated and LPS-treated blood (Fig. 5A and B).

\section{MPs are the primary source of TF in PFP from LPS-treated blood}

To investigate the contribution of MPs to thrombin generation in our in vitro model, we analyzed the effect of depleting MPs by centrifugation at 20,000 $\mathrm{g}$ for $15 \mathrm{~min}$. This procedure was designed to remove large MPs but not small MPs. We found that the LT of PFP from both untreated and LPS-treated blood was prolonged in plasma depleted of MPs (Fig. 6A). The shorter LT observed in MP-depleted PFP derived from LPS-treated blood compared with MPdepleted PFP derived from untreated blood is likely due to the presence of residual, small TFpositive MPs in the plasma from LPS-treated blood. Next, we directly measured the TF activity of MPs isolated from untreated and LPS-treated whole blood. LPS stimulation dramatically 
increased the level of TF activity in isolated MPs (Fig. 6B). These results suggest that MP TF activity is the major source of TF in PFP isolated from LPS stimulated blood.

Interestingly, direct stimulation of PRP with LPS for 5 hours did not change the LT or peak, suggesting that TF expression was not inducible in platelets (data not shown). In addition, we found that stimulation of PRP with a PAR-1 agonist peptide $(50 \mu \mathrm{M})$ shortened the LT ( 7 min versus $10 \mathrm{~min}$ ) and increased the peak ( $427 \mathrm{~nm}$ versus $137 \mathrm{~nm}$ ). The shortening of the LT by the PAR-1 agonist peptide was not affected by FVIIai (data not shown).

\section{Exogenous TF dose response in PFP}

We analyzed the effect of exogenous TF on thrombin generation in PFP generated from blood collected into citrate alone or citrate and CTI. Although the effect of TF on thrombin generation has previously been characterized, no studies have examined the effect of 0-1 pM TF concentrations on thrombin production. To produce a shift in LT that was similar to that observed in PFP prepared from LPS-treated blood we used low concentrations of exogenous TF. Phospholipids $(4 \mu \mathrm{M})$ were added to all the samples. Exogenous TF induced a dosedependent shortening of the LT with no change in peak thrombin in PFP from citrated blood (Fig. 7A and B). However, at high concentrations of recombinant TF (>1 pM) an increase in peak was observed (data not shown). In blood anticoagulated with citrate and CTI exogenous TF changed both the LT and the peak thrombin (Fig. 7C and D). We also found that exogenous TF changed the LT and peak in factor XI-deficient plasma and that the effect on peak was lost upon addition of factor XI (final concentration $4 \mu \mathrm{g} / \mathrm{mL}$ ) to the factor XI-deficient plasma (Table). These results indicate that TF can affect the peak of thrombin generation when the intrinsic pathway is inhibited or deficient.

\section{Discussion}

The goal of this study was to determine if the CAT assay could be used to detect endogenous $\mathrm{TF}$ activity in plasma. Normally, the CAT assay uses recombinant TF to initiate coagulation and has been used extensively to measure thrombin generation in a variety of clinical samples. We found that the CAT assay could be used to measure increased levels of endogenous TF in both PRP and PFP prepared from citrated blood stimulated with LPS. The presence of TF shortened the LT but did not affect the peak of thrombin generation. Similar results were observed when low levels of recombinant TF were added to PFP prepared from unstimulated blood. Based on our studies with exogenous TF we estimate that PFP from LPS-treated blood contains $<0.2 \mathrm{pM}$ of TF. These results indicate that low levels of endogenous TF contribute to the initiation of clotting without significantly affecting the amount of thrombin generated in citrated plasma.

We used LPS-treated whole blood as a model system to generate endogenous TF. LPS is a potent inducer of TF expression in monocytes (4) (9-10). We found that LPS induced a dosedependent shortening of the LT in both PRP and PFP. Depletion of the MPs by centrifugation significantly prolonged the LT in PFP prepared from LPS-treated blood. In addition, isolated MPs from LPS-treated blood contained TF activity. These results suggest that MPs are a major source of TF in LPS-treated blood. A recent study used the CAT assay to measure the procoagulant activity of MPs derived from patients with recurrent thrombosis (31). Interestingly, the LT in PFP from blood incubated without LPS for 5 hours was also prolonged by depletion of the MPs, suggesting that small amounts of MPs generated under these conditions initiate the clotting reaction. LPS induces the release of TF-positive MPs from monocytes and these MPs can bind to activated platelets (43). Platelets have also been reported to express TF, although this is controversial (44-47). Therefore, TF-positive MPs observed in our study could be derived from monocytes and/or platelets. We found that direct stimulation of PRP did not shorten the LT, suggesting that either direct stimulation of platelets with LPS 
does not induce TF expression or that the levels of TF generated from platelets are below the detectable threshold of the CAT assay. Although we cannot exclude the possibility that other factors in LPS-treated whole blood induce TF expression in platelets, our results suggest that LPS stimulation of whole blood induces TF expression in monocytes and that these cells release TF-positive MPs that are present in both PRP and PFP.

We found that the LT observed in PRP was shorter than the LT of PFP prepared from LPStreated blood. In addition, the peak of thrombin generation was much higher in PRP compared with PFP. We suspected that this was due, in part, to a limited amount of phospholipid surface in the PFP. Indeed, consistent with other studies, addition of exogenous phospholipids shortened the LT and increased the peak of thrombin generation (36) (48). A MP reagent containing phospholipids has been developed to measure the procoagulant activity of MPs in plasma. However, we found that the addition of exogenous phospholipids dramatically reduced the difference in LT between PFP prepared from untreated versus LPS-treated blood, suggesting that this reagent may reduce the TF-dependence of the assay.

The intrinsic coagulation pathway is involved in the amplification of thrombin generation during blood coagulation. Collecting blood into tubes containing citrate alone is associated with activation of factor XII (41). We investigated the role of factor XIIa in thrombin generation by comparing results using PRP and PFP prepared from blood collected into citrate alone or citrate plus CTI. We found that inhibition of factor XIIa significantly prolonged the LT and decreased the peak in PRP and PFP prepared from untreated blood. CTI also decreased the peak thrombin but did not significantly prolong the LT in PRP and PFP from LPS-treated blood. Similarly, CTI prolonged the LT at low doses of exogenous TF (0.025-0.1 pM) but not at a high dose of TF $(0.2 \mathrm{pM})$. Consistent with our results, several studies showed that factor $\mathrm{XI}$ contributes to thrombin generation when low concentrations of recombinant TF are used in the CAT assay (41) (49) (50). These results indicate that factor XIIa plays a major role initiating and amplifying the coagulation cascade in untreated blood that contains very low levels of TF but contributes less to the initiation phase of clotting in the presence of high levels of TF. Interestingly, low levels of exogenous TF affected the LT but not the peak in citrated plasma but affected the LT and the peak in citrated plasma with CTI. Similarly, low levels of exogenous TF shortened the LT and increased the peak in factor XI-deficient plasma but not in factor XI-deficient plasma with factor XIa. These results support the notion that the major role of TF in blood coagulation is to initiate the cascade and that it has a minimal role in amplification of thrombin generation when the intrinsic pathway is not inhibited. Nevertheless, there are several disadvantages to using CTI: it is expensive; it requires special collection of the blood; and it dramatically reduces the amounts of thrombin generated in the CAT assay.

The strength of the CAT assay is the measurement of thrombin generation in native plasma. One limitation of the CAT assay is that it is affected by changes in the levels of clotting factors. For instance, levels of clotting factors decrease in patients with a consumptive coagulopathy and this could lead to a decrease in thrombin generation even in the presence of increased TF levels. One study showed that sepsis patients had prolonged LT and decreased peak thrombin compared with controls due to decreased levels of clotting factors (51). Our studies indicate that the CAT assay can detect levels of endogenous TF in plasma. However, there are several factors to consider when using the CAT assay. Inhibition of FXIIa with CTI prolongs the LT and makes thrombin generation more dependent on the presence of TF. Addition of exogenous phospholipids increases thrombin generation by providing a thrombogenic surface but may decrease the TF-dependence of the reaction. Factors other than TF can affect thrombin generation and therefore CAT assays should be performed in the presence and absence of an inhibitor of TF to determine the contribution of TF to thrombin generation. Despite the positive results from this LPS whole blood model, further studies are required to determine if the CAT assay can be used to detect elevated levels of MP TF activity in clinical samples. 


\section{Acknowledgments}

We would like to thank Drs Monroe, Key and Kasthuri for critical reading of the manuscript. This work was supported by grants from the National Institutes of Health HL48872 (N.M.) and K01 AR051021 (A.W.). Dr Wolberg also received funding from the Gustavus and Louise Pfeiffer Foundation, the National Hemophilia Foundation, Novo Nordisk and the UNC Institute on Aging.

\section{Reference List}

(1). Mackman N. Role of tissue factor in hemostasis, thrombosis, and vascular development. Arterioscler Thromb Vasc Biol 2004;24(6):1015-1022. [PubMed: 15117736]

(2). Edgington TS, Mackman N, Brand K, et al. The structural biology of expression and function of tissue factor. Thromb Haemost 1991;66:67-79. [PubMed: 1833852]

(3). Nemerson Y. The tissue factor pathway of blood coagulation. Semin Hematol 1992;29:170-176. [PubMed: 1641663]

(4). Drake TA, Morrissey JH, Edgington TS. Selective cellular expression of tissue factor in human tissues. Implications for disorders of hemostasis and thrombosis. Am J Pathol 1989;134:1087-1097. [PubMed: 2719077]

(5). Franco RF, de Jonge E, Dekkers PEP, et al. The in vivo kinetics of tissue factor messenger RNA expression during human endotoxemia: relationship with activation of coagulation. Blood 2000;96:554-559. [PubMed: 10887118]

(6). Morrissey, JH.; Drake, TA. Procoagulant response of the endothelium and monocytes. In: Schlag, G.; Redl, H., editors. Pathophysiology of shock, sepsis and organ failure. Springer-Verlag; Berlin, New York: 1993. p. 564-574.

(7). Osterud B, Flaegstad T. Increased tissue thromboplastin activity in monocytes of patients with meningococcal infection: Related to an unfavorable prognosis. Thromb Haemost 1983;49:5-7. [PubMed: 6845273]

(8). Almdahl SM, Osterud B. Experimental gram-negative septicemia: Thromboplastin generation in mononuclear phagocytes from different anatomical sites. Thromb Res 1987;47:37-46. [PubMed: 3660344]

(9). Gregory SA, Morrissey JH, Edgington TS. Regulation of tissue factor gene expression in the monocyte procoagulant response to endotoxin. Mol Cell Biol 1989;9:2752-2755. [PubMed: 2503712]

(10). Osterud B, Bjorklid E. The production and availability of tissue thromboplastin in cellular populations of whole blood exposed to various concentrations of endotoxin. Scand J Haematol 1982;29:175-184. [PubMed: 6813961]

(11). Brand K, Fowler BJ, Edgington TS, et al. Tissue factor mRNA in THP-1 monocytic cells is regulated at both transcriptional and posttranscriptional levels in response to lipopolysaccharide. Mol Cell Biol 1991;11:4732-4738. [PubMed: 1875949]

(12). Mackman N, Brand K, Edgington TS. Lipopolysaccharide-mediated transcriptional activation of the human tissue factor gene in THP-1 monocytic cells requires both activator protein 1 and nuclear factor $\square$ B binding sites. J Exp Med 1991;174:1517-1526. [PubMed: 1744583]

(13). Morel O, Toti F, Hugel B, et al. Procoagulant microparticles: disrupting the vascular homeostasis equation? Arterioscler Thromb Vasc Biol 2006;26(12):2594-2604. [PubMed: 16990554]

(14). Nieuwland R, Berckmans RJ, Rotteveel-Eijkman RC, et al. Cell-derived microparticles generated in patients during cardiopulmonary bypass are highly procoagulant. Circulation 1997;96:35343541. [PubMed: 9396452]

(15). Diamant M, Nieuwland R, Pablo RF, et al. Elevated numbers of tissue-factor exposing microparticles correlate with components of the metabolic syndrome in uncomplicated type 2 diabetes mellitus. Circulation 2002;106(19):2442-2447. [PubMed: 12417540]

(16). Aras O, Shet A, Bach RR, et al. Induction of microparticle- and cell-associated intravascular tissue factor in human endotoxemia. Blood 2004;103:4545-4553. [PubMed: 14988149]

(17). Shet AS, Aras O, Gupta K, et al. Sickle blood contains tissue factor-positive microparticles derived from endothelial cells and monocytes. Blood 2003;102(7):2678-2683. [PubMed: 12805058] 
(18). Hron G, Kollars M, Weber H, et al. Tissue factor-positive microparticles: cellular origin and association with coagulation activation in patients with colorectal cancer. Thromb Haemost 2007;97 (1):119-123. [PubMed: 17200778]

(19). Tesselaar ME, Romijn FP, Van Der Linden IK, et al. Microparticle-associated tissue factor activity: a link between cancer and thrombosis? J Thromb Haemost Mar;2007 5(3):520-7. [PubMed: 17166244]

(20). Biro E, Sturk-Maquelin KN, Vogel GM, et al. Human cell-derived microparticles promote thrombus formation in vivo in a tissue factor-dependent manner. J Thromb Haemost 2003;1(12):2561-2568. [PubMed: 14738565]

(21). Mallat Z, Hugel B, Ohan J, et al. Shed membrane microparticles with procoagulant potential in human atherosclerotic plaques: a role for apoptosis in plaque thrombogenicity. Circulation 1999;99 (3):348-353. [PubMed: 9918520]

(22). Sommeijer DW, Hansen HR, van Oerle R, et al. Sobuble tissue factor is a candidate marker for progression of microvascular disease in patient with Type 2 diabetes. J Thromb Haemost 2006;4 (3):574-580. [PubMed: 16371118]

(23). Langer F, Chun FK, Amerkhosravi A, et al. Plasma tissue factor antigen in localized prostate cancer: distribution,clinical significance and correlation with haemostatic activation markers. Thromb Haemost 2007;97(3):464-470. [PubMed: 17334515]

(24). Misumi K, Ogawa H, Yasue H, et al. Comparison of plasma tissue factor levels in unstable and stable angina pectoris. Am J Cardiol 1998;81:22-26. [PubMed: 9462600]

(25). Soejima H, Ogawa H, Yasue H, et al. Plasma tissue factor pathway inhibitor and tissue factor antigen levels after administration of heparin in patients with angina pectoris. Thromb Haemost 1999;93:17-25.

(26). Soejima H, Ogawa H, Yasue H, et al. Heightened tissue factor associated with tissue factor pathway inhibitor and prognosis in patients with unstable angina. Circulation 1999;99:2908-2913. [PubMed: 10359735]

(27). Shah MD, Bergeron AL, Dong JF, et al. Flow cytometric measurement of micropartilces: pitfalls and protocol modifications. Platelets 2008;19(5):365-372. [PubMed: 18791943]

(28). Khorana A, Francis CW, Menzies KE, et al. Plasma tissue factor may be predictive of venous thromboembolism in pancreatic cancer. J Thromb Haemost 2008;6:1983-1985. [PubMed: 18795992]

(29). Hemker HC, Willems GM, Beguin S. A computer assisted method to obtain the prothrombin activation velocity in whole plasma independent of thrombin decay processes. Thromb Haemost 1986;56(1):9-17. [PubMed: 2430342]

(30). Duchemin J, Pan-Petesch B, Arnaud B, et al. Influence of coagulation factors and tissue factor concentration on the thrombin generation test in plasma. Thromb Haemost 2008;99:767-773. [PubMed: 18392335]

(31). Bidot L, Jy W, Bidot C, et al. Microparticle-mediated thrombin generation assay: increased activity in patients with recurrent thrombosis. J Thromb Haemost 2008;6:913-919. [PubMed: 18363818]

(32). Dielis AW, Castoldi E, Spronk HM, et al. Coagulation factors and the protein C system as determinants of thrombin generation in a normal population. J Thromb Haemost 2008;6:125-131. [PubMed: 17988231]

(33). Hemker HC, Giesen P, Aldieri R, et al. The calibrated automated thrombogram (CAT): a universal routine test for hyper- and hypocoagulability. Pathophysiol Haemost Thromb 2002;32(56):249253. [PubMed: 13679651]

(34). Gatt A, van Veen JJ, Woolley AM, et al. Thrombin generation assays are superior to traditional tests in assessing anticoagulation reversal in vitro. Thromb Haemost 2008;100(2):350-355. [PubMed: 18690358]

(35). Gerotziafas GT, Depasse F, Chakroun T, et al. Comparison of the effect of fondaparinux and enoxaparin on thrombin generation during in-vitro clotting of whole blood and platelet-rich plasma. Blood Coagul Fibrinolysis 2004;(15):149-156. [PubMed: 15091002]

(36). Livnat T, Zivelin A, Martinowitz O, et al. Prerequisites for recombinant factor VIIa-induced thrombin generation in plasmas deficient in factors VIII, IX or XI. J Thromb Haemost 2006;4(1): 192-200. [PubMed: 16409469] 
(37). Koestenberger M, Cvirn G, Nagel B, et al. Thrombin generation determined by calibrated automated thrombography (CAT) in pediatric patients with congenital heart disease. Thromb Res 2008;122:13-19. [PubMed: 17915295]

(38). Collins PW, Macchiavello LI, Lewis SJ, et al. Global tests of haemostasis in critically ill patients with severe sepsis syndrome compared to controls. Br J Haematol 2006;135:220-227. [PubMed: 17010048]

(39). ten Cate-Hoek AJ, Dielis AW, Spronk HM, et al. Thrombin generation in patients after acute deepvein thrombosis. Thromb Haemost 2008;100:240-245. [PubMed: 18690343]

(40). Dargaud Y, Trzeciak MC, Bordet JC, et al. Use of calibrated automated thrombinography +/thrombomodulin to recognise the prothrombotic phenotype. Thromb Haemost 2006;96:562-567. [PubMed: 17080211]

(41). Luddington R, Baglin T. Clinical measurement of thrombin generation by calibrated automated thrombography requires contact factor inhibition. J Thromb Haemost 2008;2(11):1954-1959. [PubMed: 15550027]

(42). Carson SD, Ross SE, Bach R, et al. An inhibitory monoclonal antibody against human tissue factor. Blood 1987;70:490-493. [PubMed: 3607285]

(43). Del Conde I, Shrimpton CN, Thiagarajan P, et al. Tissue-factor-bearing microvesicles arise from lipid rafts and fuse with activated platelets to initiate coagulation. Blood 2005;106(5):1604-1611. [PubMed: 15741221]

(44). Zillmann A, Luther T, Muller I, et al. Platelet-associated tissue factor contributes to the collagentriggered activation of blood coagulation. Biochem Biophys Res Commun 2001;281(2):603-609. [PubMed: 11181090]

(45). Butenas S, Bouchard BA, Brummel-Ziedins KE, et al. Tissue factor activity in whole blood. Blood 2005;105(7):2764-2770. [PubMed: 15604222]

(46). Panes O, Matus V, Saez CG, et al. Human platelets synthesize and express functional tissue factor. Blood 2007;109:5242-5250. [PubMed: 17347408]

(47). Schwertz H, Tolley ND, Foulks JM, et al. Signal-dependent splicing of tissue factor premRNA modulates the thrombogenicity of human platelets. J Exp Med 2006;203(11):2433-2440. [PubMed: 17060476]

(48). Butenas S, Branda RF, van't Veer C, et al. Platelets and phospholipids in tissue factor-initiated thrombin generation. Thromb Haemost 2002;87(3):551-554. [PubMed: 11916097]

(49). van Veen JJ, Gatt A, Cooper P, et al. Corn trypsin inhibitor in fluorogenic thrombin-generation measurements is only necessary at low tissue factor concentrations and influences the relationship between factor VIII coagulant activity and thrombogram parameters. Blood Coagul Fibrinolysis 2008;19:183-189. [PubMed: 18388496]

(50). Keularts IMLW, Zivelin A, Seligsohn U, et al. The role of factor XI in thrombin generation induced by low concentrations of tissue factor. Thromb Haemost 2001;85:1060-1065. [PubMed: 11434685] 
A

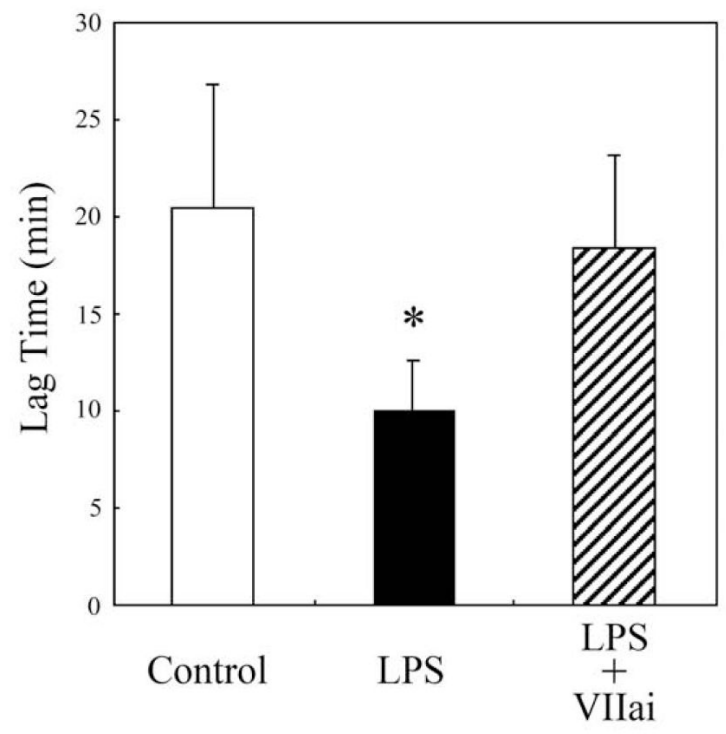

B

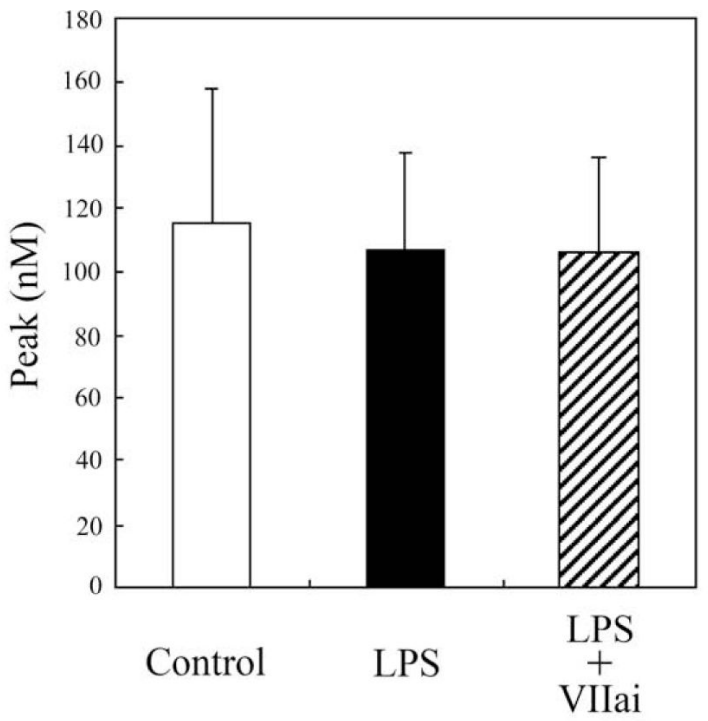

Figure 1. Thrombin generation in LPS-treated PRP

PRP was prepared from citrated human whole blood that was treated with either PBS control (white bars) or LPS $(10 \mu \mathrm{g} / \mathrm{mL})$ (black bars) for $5 \mathrm{~h}$ at $37^{\circ} \mathrm{C}$. PRP was recalcified and thrombin generation measured using the CAT assay. PRP from LPS stimulated blood was also preincubated with factor VIIai $(100 \mathrm{nM})$ (hatched bars) for $10 \mathrm{~min}$ at room temperature prior to measuring thrombin generation. Two parameters of thrombin generation are shown (mean $\pm \mathrm{SD}, \mathrm{n}=15)$ : LT (Panel A), peak (Panel B). The asterisk indicates a statistically significant difference from the control $p<0.05$. 
A

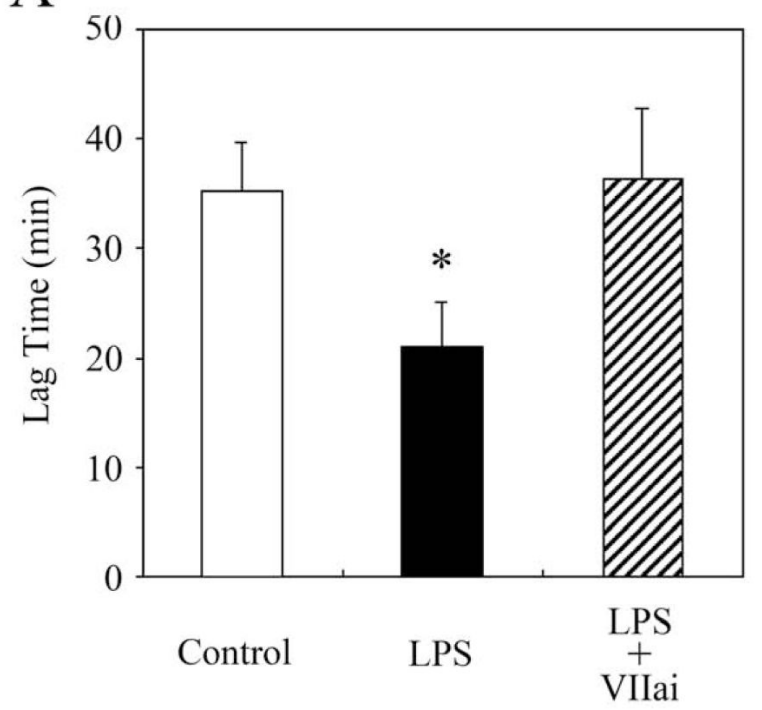

$\mathrm{B}$

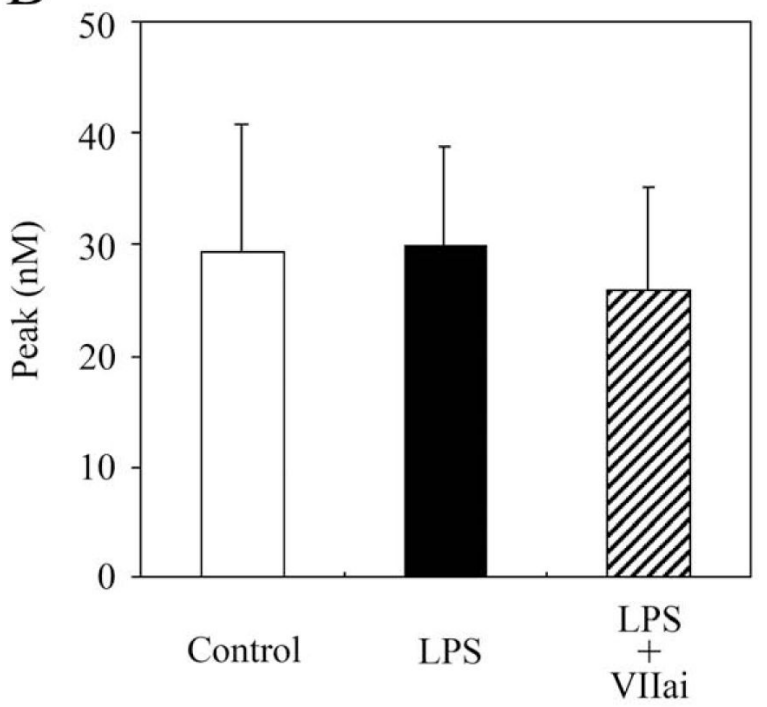

Figure 2. Thrombin generation in LPS-treated PFP

PFP was isolated from citrated human whole blood that was treated with either PBS control (white bars) or LPS $\left(10 \mu \mathrm{g} / \mathrm{mL}\right.$ ) (black bars) for $5 \mathrm{~h}$ at $37^{\circ} \mathrm{C}$. PFP was recalcified and thrombin generation measured using the CAT assay. PFP from LPS stimulated blood was also preincubated with factor VIIai $(100 \mathrm{nM}$ ) (hatched bars) for $10 \mathrm{~min}$ at room temperature prior to measuring thrombin generation. Two parameters of thrombin generation are shown (mean \pm SD, $\mathrm{n}=6$ ): LT (Panel A) and peak (Panel B). The asterisk indicates a statistically significant difference from the control $p<0.05$. 

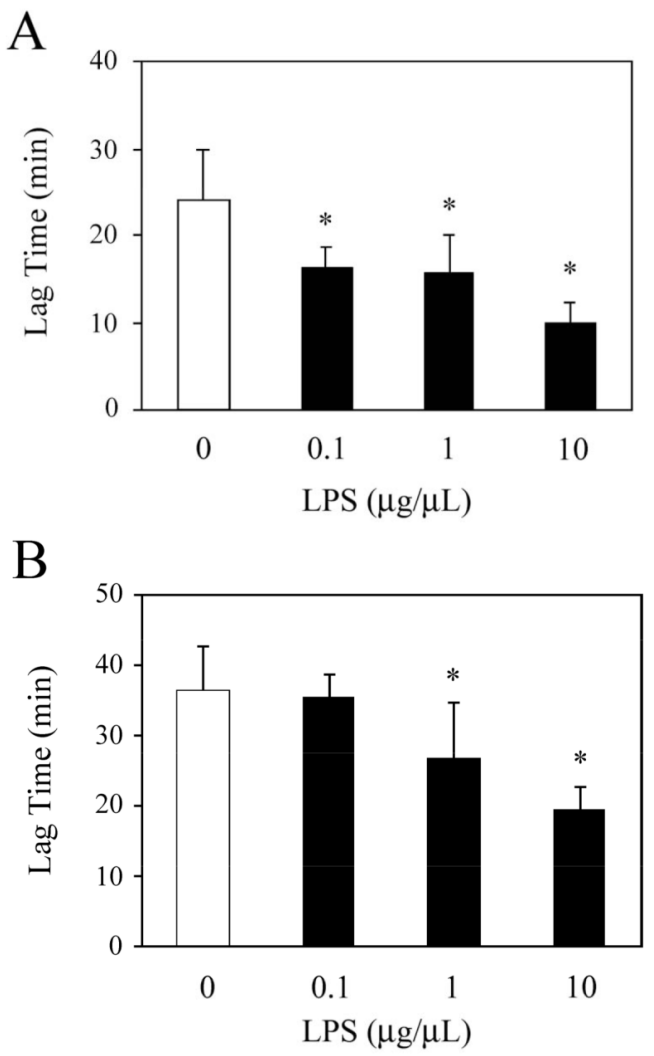

Figure 3. LPS dose response

Citrated whole blood was treated with PBS control or LPS $(0.1,1$ and $10 \mu \mathrm{g} / \mathrm{mL})$ for $5 \mathrm{~h}$ followed by preparation of PRP and PFP. Thrombin generation in PRP (Panel A) and PFP (Panel B) was measured using the CAT assay. The LT of thrombin generation is shown (mean $\pm \mathrm{SD}, \mathrm{n}=5$ ). Asterisks indicate a statistically significant difference from the control $p<0.05$. 
A

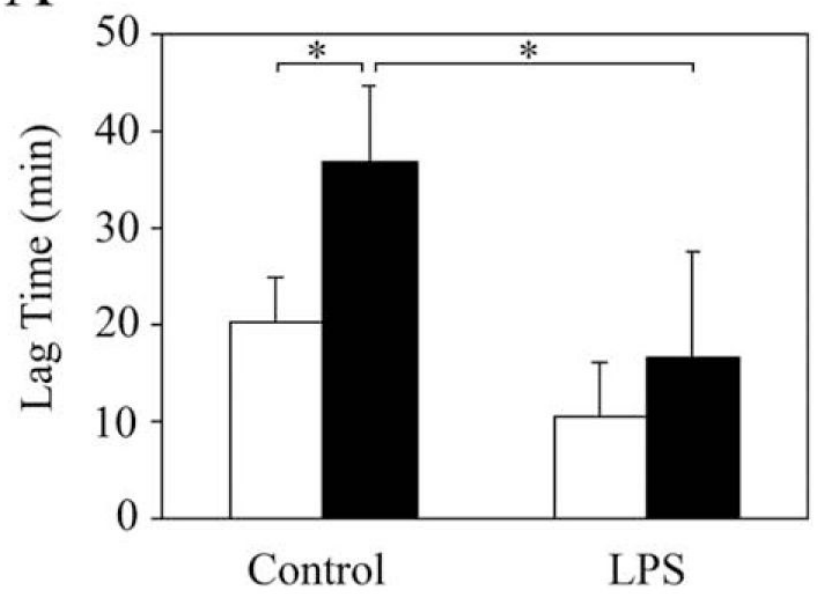

C

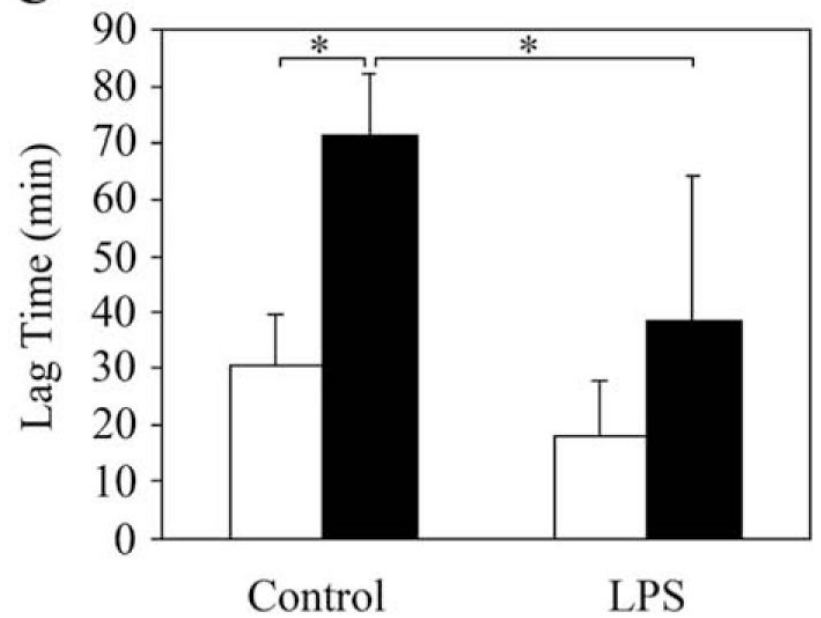

B

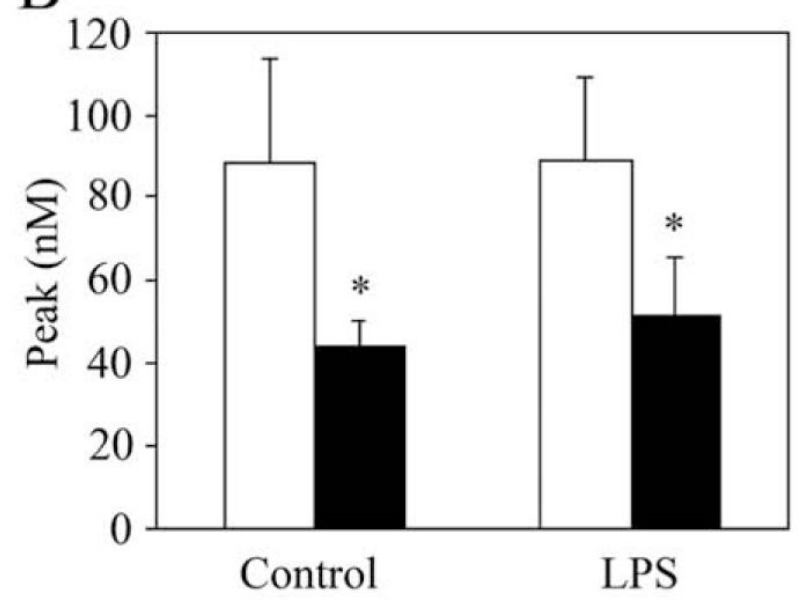

D

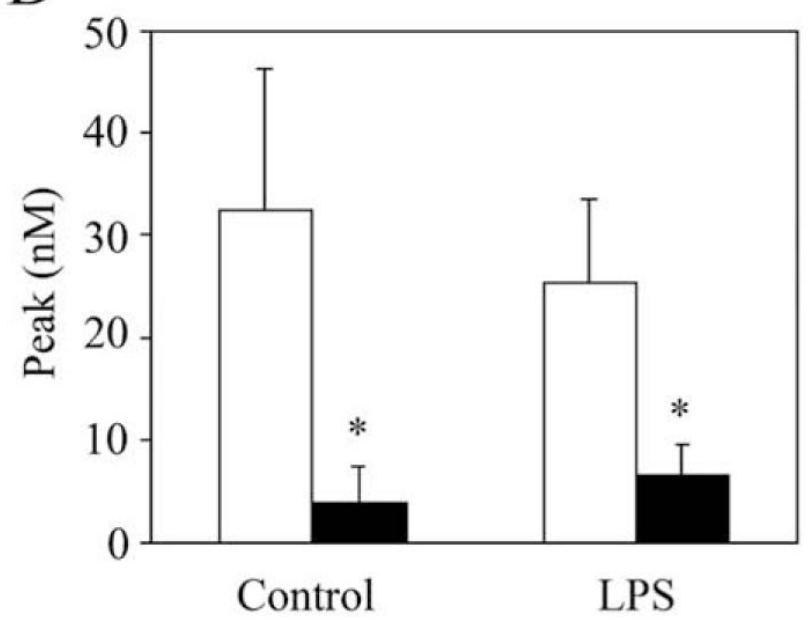

Figure 4. Role of factor XIIa in thrombin generation in PRP and PFP

Whole blood was anticoagulated with either sodium citrate alone (white Bars) or sodium citrate and CTI $(18.3 \mu \mathrm{g} / \mathrm{mL})$ (black bars) and incubated at $37^{\circ} \mathrm{C}$ for $5 \mathrm{~h}$ with or without LPS $(10 \mu \mathrm{g} /$ $\mathrm{ml}$. Thrombin generation in recalcified PRP (Panels A and B, n=4) and PFP (Panels C and D, $\mathrm{n}=4$ ) was measured using the CAT assay. Data are shown as mean \pm SD. Asterisks indicate a statistically significant difference from the control $p<0.05$. 

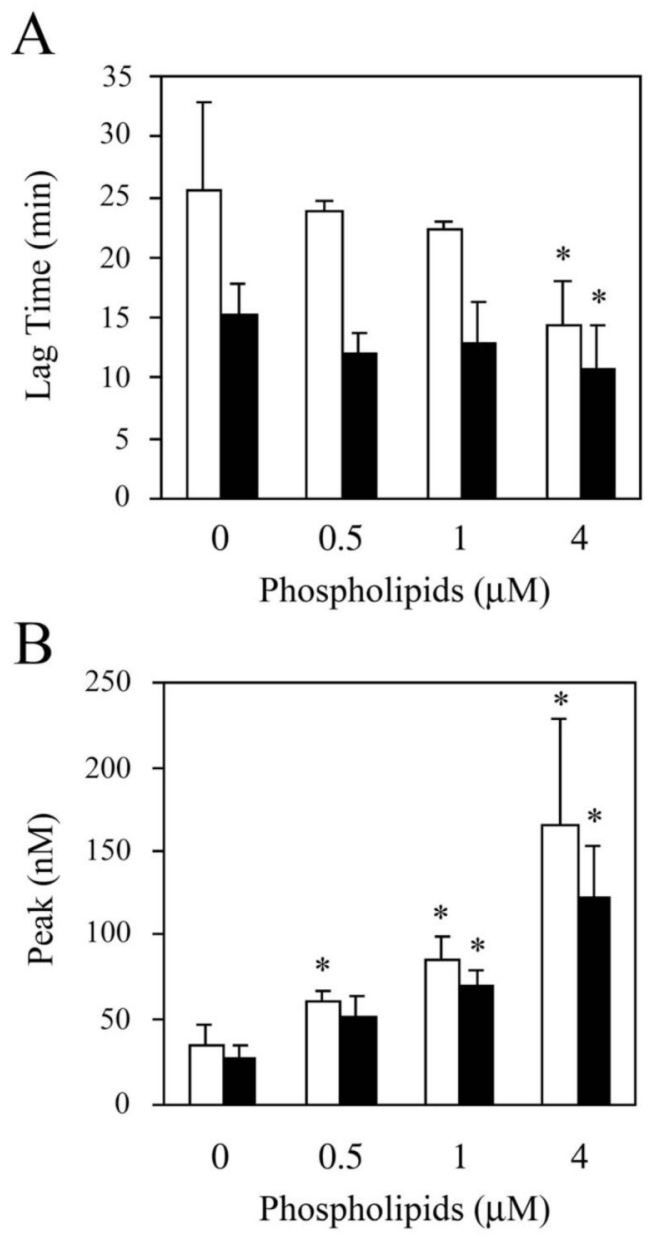

Figure 5. Effect of exogenous phospholipids on thrombin generation in PFP

PFP was prepared from whole blood with or without LPS stimulation. Phospholipids (0, 0.5, 1 and $4 \mu \mathrm{M}$ ) were added to PFP before measuring thrombin generation. Results (mean $\pm \mathrm{SD}$, $\mathrm{n}=4$ ) from PFP prepared from untreated (white bars) and LPS-treated (black bars) are shown. Asterisks indicate a statistically significant difference from the control $p<0.05$. 
A

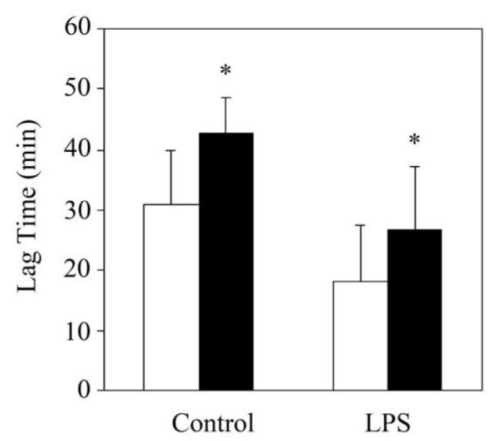

B

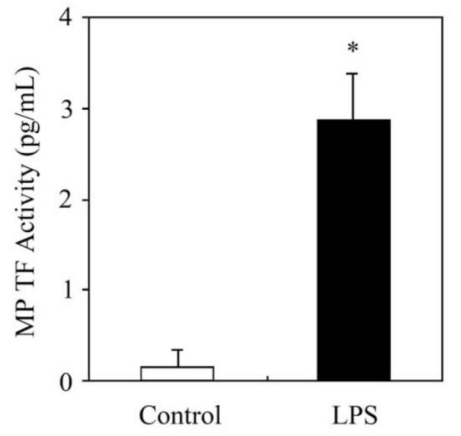

Figure 6. Role of MPs in thrombin generation in PFP from untreated and LPS-treated blood A/ Thrombin generation was measured in PFP (white bars) or PFP depleted of MPs (black bars). MPs in PFP were depleted by centrifugation at 20,000g for $20 \mathrm{~min}$. Results from PFP prepared from untreated and LPS $(10 \mu \mathrm{g} / \mathrm{ml})$-treated blood are shown as mean $\pm \mathrm{SD}, \mathrm{n}=5$ (Panel A). B/ MPs were prepared from PFP that was prepared from untreated (white bar) or LPS-treated (black bars) blood ( $\mathrm{n}=3$ ). MP TF activity was measured in a two-stage chromogenic assay. Asterisks indicate a statistically significant difference from the control $p$ $<0.05$. 

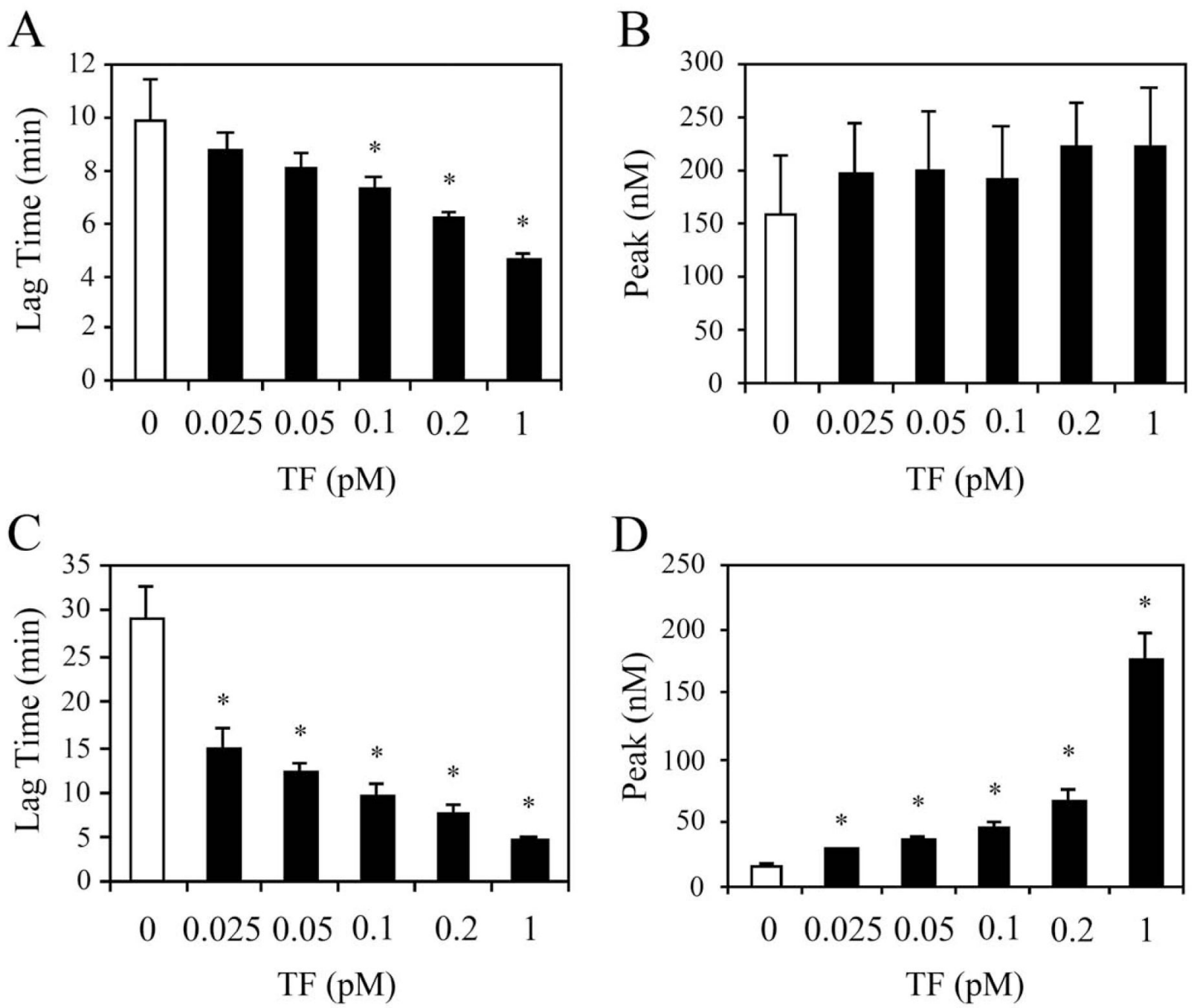

Figure 7. Effect of exogenous TF on thrombin generation in PFP with of without inhibition of FXIIa Whole blood was collected into either sodium citrate or sodium citrate and CTI and used to prepare PFP. Exogenous TF (0.025-1.0 pM) and phospholipid ( $4 \mu \mathrm{M})$ were added to PFP from citrated blood (Panels A and B) or PFP from blood anticoagulated with citrate and CTI (Panels $\mathrm{C}$ and D) and the LT and peak of thrombin generation measured. Data are shown as mean \pm $\mathrm{SD}(\mathrm{n}=3)$. Asterisks indicate a statistically significant difference from the control $p<0.05$. 
Table

Effect of exogenous TF on thrombin generation in factor XI-deficient plasma with or without factor XI.

\begin{tabular}{|c|c|c|c|c|}
\hline \multirow{2}{*}{ TF $(\mathbf{p M})$} & \multicolumn{2}{|c|}{ FXI-deficient plasma } & \multicolumn{2}{c|}{ FXI-deficient plasma + FXI } \\
\cline { 2 - 5 } & LT (min) & Peak (nM) & LT (min) & Peak (nM) \\
\hline 0 & ND & 0 & 16.7 & 147.5 \\
\hline 0.025 & ND & 0 & 10.5 & 129.5 \\
\hline 0.05 & ND & 0 & 9.8 & 132.0 \\
\hline 0.1 & 24.5 & 2.4 & 9.3 & 123.0 \\
\hline 0.2 & 12.5 & 5.8 & 8.2 & 145.5 \\
\hline 1.0 & 5.7 & 28.9 & 5.2 & 125.0 \\
\hline
\end{tabular}

ND - Not Detectable. MP reagent $(4 \mu \mathrm{M})$ was added to each reaction. Data is shown as mean of two independent experiments. 